kung. Schließlich handelt es sich hier um einen Fall, der von den politischen Eliten in den westlichen Ländern als zentral für die eigene Sicherheit verstanden wird und für dessen Lösung die internationale Gemeinschaft bereits massive Ressourcen aufgewendet hat. Wenn nun selbst dieses Land in einem $\mathrm{Zu}$ stand zurückgelassen würde, der höchstwahrscheinlich eine katastrophale Menschenrechtssituation nach sich zöge, würde nicht zuletzt die Aussicht auf ein Eingreifen externer Akteure in jenen Situationen sinken, in denen diese über keine eng verstandenen Sicherheitsinteressen verfügen. Zudem würde ein solcher Rückzug die ganz essentielle Frage aufwerfen, wie viel der „internationalen Gemeinschaft“ und vor allem den liberalen westlichen Demokratien die vielgepriesenen liberalen Werte tatsächlich wert sind. Sollte in Afghanistan also etwas zu Grabe getragen werden, dann könnte dies die westliche Überzeugung sein, dass der „liberale Moment“ aktiv durch Weltordnungspolitik getragen und unterstützt werden sollte. Er wäre dann tatsächlich nur ein „Moment“ gewesen.

\title{
Afghanistan - Ein langer Weg zur Rechtsstaatlichkeit
}

\author{
Julia Pfeiffer*
}

\begin{abstract}
Since 2001 the international community supports the Afghan state in building the rule of law in the country. Although some progress has been made, the rule of law is still weak. However, as the paper shows, the situation of decreasing security and lack of the rule of law are interrelated and affect each other in a negative way. Therefore, strengthening the rule of law should be the priority in the ongoing state-building process. As long as the Afghan government cannot provide a stable and secure environment based on the principles of the rule of law, reconciliation and peace cannot be achieved. Afghan authorities must finally start to accept and implement the concepts of rule of law by ceasing injustice, impunity and corruption throughout the country. The new "Prioritization and Implementation Plan", which the Afghan government presented on the International Kabul Conference in July 2010, may be a first step in this direction.
\end{abstract}

Keywords: Afghanistan, state-building, rule of law and stability, judicial reform

Afghanistan, State-Building, Rechtsstaatlichkeit und Stabilität, Justizreform

\section{Einleitung}

$\mathrm{Z}$ um Wiederaufbau Afghanistans, der seit nunmehr fast neun Jahren andauert, gehört auch die Schaffung eines stabilen Rechtsstaats. Dieses Ziel ist auch in der Präambel der neuen Verfassung Afghanistans von 2004 vorgesehen: „Wir das Volk von Afghanistan, haben (...) zur Errichtung einer Zivilgesellschaft (...) basierend auf Rechtsstaatlichkeit, sozialer Gerechtigkeit, dem Schutz der Menschenrechte und der Menschenwürde (...) diese Verfassung (...) verabschiedet.“ Auch wenn das Rechtsstaatsprinzip im Verfassungstext selbst nicht ausdrücklich genannt wird, so kann es aus verschiedenen Vorschriften abgeleitet werden.

Der folgende Artikel befasst sich mit der Frage, in welchem Umfang Rechtsstaatlichkeit in Afghanistan vorhanden ist und führt weiter aus, inwieweit ein Zusammenhang zwischen Rechtsstaatlichkeit, Stabilität und Sicherheit hergestellt werden kann. Beleuchtet werden außerdem die letzten Entwicklungen beim Staatsaufbau in Afghanistan seit dem Sommer 2010.

\section{Rechtsstaatlichkeit in Afghanistan?}

Rechtsstaatlichkeit setzt sich aus fünf Prinzipien zusammen: Rechtsbindung der Gewalten, Gleichheit vor dem Gesetz, Ge- währleistung der öffentlichen Ordnung, eine effiziente und unabhängige Justiz sowie Achtung der Menschenrechte. ${ }^{1}$

Obwohl die Gesetzesbindung der Exekutive in der afghanischen Verfassung (AV) in den Artikeln 50, 63, 76 und 77 vorgesehen ist, setzt sich die Exekutive immer wieder über dieses Prinzip hinweg. So geschehen zum Beispiel beim Misstrauensvotum gegen den ehemaligen Außenminister Spanta aus dem Jahr 2007, das von Präsident Karzai ignoriert wurde. ${ }^{2}$ Spanta blieb bis Januar 2010 Außenminister.

Die Gerichte sollen laut Artikel 130 der afghanischen Verfassung ihre Urteile auf Grundlage der Verfassung und Gesetze fällen. Dies geschieht in vielen Fällen jedoch nicht. Der Grund hierfür ist insbesondere die weit verbreitete Korruption in der Richter- und Staatsanwaltschaft. ${ }^{3}$ Parteilichkeit und Bestech-

* Die Autorin ist Mitarbeiterin der Afghanistan-Projekte des Max-Planck-Instituts für ausländisches öffentliches Recht und Völkerrecht (jpfeiffe@mpil.de). Die im Text geäußerte Meinung ist allein die der Autorin.

1 Rachel Kleinfeld "Competing Definitions of the Rule of Law" in Thomas Carothers, "Promoting the Rule of Law Abroad - In Search of Knowledge", Carnegie Endowment for International Peace, Washington D.C., 2006, S. 31 ff. Im Folgenden stützt sich die Autorin auf diese Ansätze. Zur akademischen Diskussion in diesem Bereich siehe Kleinfeld S. 32, Fn 10

2 Siehe Ramin Moschtaghi "Aktuelle Probleme beim Rechtsstaatsaufbau in Afghanistan: Das Gutachten des Obersten Gerichtshofs zum Misstrauensantrag des Unterhauses gegen den Außenminister" in ZaöRV 68 (2002) 2, S. 509 ff.

3 UNDOC Report: Corruption in Afghanistan - Bribery as reported by the Victims, January 2010, S. 27f. (http://www.reliefweb.int/rw/RWFiles2010. nsf/FilesByRWDocUnidFilename/FBUO-7ZUKNH-full_report.pdf/\$File/ full_report.pdf (10. September 2010). 
lichkeit der Judikative haben negative Auswirkungen auf das Prinzip der Gleichheit vor dem Gesetz, welches impliziert, dass alle Bürger, unabhängig von Herkunft, Geschlecht oder sozialem Stand die gleichen Rechte, Pflichten und Konsequenzen erfahren. In Afghanistan werden Entscheidungen oftmals zu Gunsten der finanziell bessergestellten und einflussreicheren Partei und ohne Berücksichtigung der tatsächlichen Rechtslage getroffen. Obwohl der Gleichheitssatz in Artikel 22 AV verankert ist, konnte außerdem eine tatsächliche Gleichstellung von Mann und Frau bislang nicht erreicht werden. ${ }^{4}$ Das Prinzip der Gleichheit vor dem Gesetz gilt in Afghanistan faktisch auch nicht für Straftäter, die über großen Einfluss oder gute Kontakte verfügen; sie bleiben oftmals straffrei oder werden bisweilen vom Präsidenten nachträglich begnadigt. ${ }^{5}$

Auch Verbrechen, die mit dem nun fast 30 Jahre dauernden Konflikt in Zusammenhang stehen, wurden bislang nicht aufgearbeitet. Zwar wurde ein „Action Plan for Peace and Reconciliation in Afghanistan“ entworfen, der bis Ende 2008 hätte umgesetzt werden müssen. Er ist aber niemals implementiert worden. Es ist unklar, ob der Aktionsplan überhaupt noch in Kraft ist. Der Prozess der Übergangsjustiz wurde außerdem durch den Erlass des Amnestie-Gesetzes erschwert, das rechtliche Konsequenzen für diese Verbrechen verhindert, solange die Opfer selbst keine rechtlichen Schritte unternehmen. ${ }^{6}$

Jedes rechtsstaatlich handelnde System sollte außerdem den Schutz von Leben und Eigentum eines jeden Bürgers sowie die Achtung der Menschenrechte gewährleisten. ${ }^{7}$ In der Praxis wird dieses Prinzip hauptsächlich durch die Polizei umgesetzt. ${ }^{8}$ Sie kann ihre Aufgabe jedoch nur in Zusammenarbeit mit der Justiz erfüllen, die für die Verhängung von Strafen zuständig ist und somit einen wesentlichen Beitrag zur Herstellung der öffentlichen Ordnung leisten sollte. ${ }^{9}$ Die Situation in Afghanistan ist auch hier ernüchternd. Auch wenn in den letzten Jahren verstärkt Menschenrechtsschulungen für die Polizei durchgeführt wurden, hat sich diese Institution mittlerweile dennoch zu einer eigenständigen Macht entwickelt, deren Handeln von Korruption und Gewalt gegenüber der Bevölkerung geprägt ist. ${ }^{10}$ Wie oben bereits angesprochen, leistet auch die Justiz aufgrund von Bestechlichkeit und Korruption bislang keine ausreichende Unterstützung bei der Herstellung der öffentlichen Ordnung. Ein Grund für die Bestechlichkeit ist unter anderem die schlechte Bezahlung des Justizpersonals; in manchen Provinzen verdienen Justizangestellte gerade einmal

4 Zur Situation der Frauen in Afghanistan s. z.B. AIHRC „Evaluation Report on General Situation of Women in Afghanistan“, http://www.aihrc.org.af/rep_eng wom situation 8 march.htm (10. September 2010).

5 Stephen Carter, Kate Clark „Snakes and Scorpions - Justice and Stability in Afghanistan", Independent Report prepared for The Office of the High Commissioner for Human Rights, Kabul, Afghanistan, May 2010, S. 42 ff

6 Siehe auch den Bericht der Afghanistan Research and Evaluation Unit "The State of Transitional Justice in Afghanistan", abrufbar unter www.areu.org.af.

Kleinfeld (Fn 1), S. 40.

8 So unterscheiden sowohl EU als auch USA ihre Projekte beim Staatsaufbau zwischen Rechtsstaatsprojekten, die ausschließlich die Justiz unterstützen, und Polizeitrainings, welche die Öffentliche Ordnung gewährleisten sollen. Diese Schulungen beinhalten jedoch auch Rechtsstaatskomponenten, wie z.B. Menschenrechtstrainings.

9 Kleinfeld (Fn 1), S. 41.

10 Eine Übersicht über derartige Vorfälle ist zu finden in Royal United Service Institute for Defence and Security Studies (RUSI) "Reforming the Afghan National Police", Chapter 1.2 "Failings of the ANP", S. $6 \mathrm{ff}$ (http://www.rusi. org/downloads/assets/ANP_Nov09.pdf, 10. September 2010). zwischen 80 und 150 US-Dollar im Monat. ${ }^{11}$ Um die Effizienz der Justiz sicherzustellen, bedarf es neben einer adäquaten Bezahlung außerdem einer Verbesserung von Infrastruktur und Ausstattung. Hierzu gehören insbesondere der Zugang zu aktuellen Gesetzestexten und die Bekanntmachung von neuen Gesetzen bzw. Gesetzesänderungen. Dies wird in Afghanistan nur schleppend umgesetzt und viele Richter in den Provinzen verfügen nicht über aktuelle Gesetzessammlungen. ${ }^{12}$ Des Weiteren ist für die Bürger in vielen Gebieten der Zugang zu den formellen Institutionen der Justiz schwierig bis unmöglich. Um Streitigkeiten beizulegen, bedienen sich die meisten Afghanen daher informeller Mechanismen, wie Shuras oder Jirgas. ${ }^{13}$

Die Unabhängigkeit der Justiz ist in Artikel 116 der afghanischen Verfassung vorgegeben - und zwar sowohl die institutionelle als auch die individuelle. Dennoch scheinen einige Richter allein aufgrund „guter Beziehungen“ ernannt worden zu sein. ${ }^{14}$ Für die individuelle Unabhängigkeit ist die persönliche Sicherheit des Justizpersonals eine wichtige Voraussetzung, sodass Entscheidungen frei von äußeren Beeinflussungen und allein auf Grundlage des Gesetzes getroffen werden können. Die persönliche Sicherheit für Justizangestellte kann jedoch momentan nicht ausreichend gewährleistet werden ${ }^{15}$ So kommt es nicht selten zu unfairen Entscheidungen. ${ }^{16}$

Ein weiteres Prinzip der Rechtsstaatlichkeit ist schließlich die Beachtung von Grund- und Menschenrechten durch die staatlichen Institutionen. In der afghanischen Verfassung ist der Menschenrechtsschutz in Artikel 6 verankert, Artikel 7 AV verpflichtet den Staat außerdem, die ratifizierten internationalen Verträge sowie die Allgemeine Erklärung der Menschenrechte zu achten. Die afghanische Verfassung beinhaltet darüber hinaus einen umfassenden Grundrechtskatalog, der die meisten auch international geschützten Menschenrechte enthält. Auch wenn dies in der Theorie fortschrittlich klingt, so ist wiederum die praktische Umsetzung mangelhaft. ${ }^{17}$ Das Recht auf Bildung (Artikel $43 \mathrm{AV}$ ) wird beispielsweise oftmals nicht umgesetzt, da es auf allen Bildungsebenen - insbesondere in den Provinzen - an entsprechenden Einrichtungen fehlt. Des Weiteren ist der Zugang zu medizinischer Versorgung oder sonstigen sozialen Dienstleistungen in weiten Teilen des Landes nicht gewährleistet. Viele Bürger müssen daher für die Inanspruchnahme staatlicher Leistungen oftmals lange Wege zurücklegen, weil

11 TLO Research Report: „Linkages between State and Non-State Justice Systems in Eastern Afghanistan“, Mai 2009, S. 10. Das Gehalt für Polizisten wurde vor Kurzem erhöht. Je nach Dienstgrad verdient ein Polizist nun zwischen 100 und 325 US \$, was gerade genug ist, um eine Familie zu ernähren (Quelle: Auswärtiges Amt, http://www.auswaertiges-amt.de/diplo/de/Aussenpolitik/ RegionaleSchwerpunkte/AfghanistanZentralasien/AktuelleArtikel/081010 LOTFA-10Mio,navCtx=53350.html; 10. September 2010).

12 Afghanistan Human Development Report 2007 „Bridging Modernity and Tradition - Rule of Law and the Search for Justice”, S. 71, Schaubild 4.1.

13 Es gibt unterschiedliche Zahlen darüber, wie viele Streitigkeiten in formellen und wie viele in informellen Institutionen entschieden werden. Es ist aber zu vermuten, dass wohl mehr als die Hälfte aller Fälle nicht vor ein formelles Gericht gebracht werden.

14 Afghanistan Human Development Report 2007 (Fn 12) S. 72.

15 Zum Zeitpunkt des Entstehens dieses Artikels lebten beispielweise die sieben Richter der Provinzhauptstadt Kandahar permanent im Gerichtsgebäude. Die meisten von ihnen konnten das Gebäude niemals verlassen, zwei immerhin gelegentlich, da sie in der Bevölkerung und auch bei den Taliban den Ruf hatten, nicht korrupt zu sein.

16 Siehe Afghanistan Human Development Report 2007 (Fn 12), S. 72.

17 Eine gute Übersicht über die Menschenrechtslage in Afghanistan bieten die Publikationen der AIHRC unter http://www.aihrc.org.af/. 
Infrastrukturen fehlen. ${ }^{18}$ Solche Reisen sind aufgrund der schlechten Sicherheitslage häufig mit hohen Risiken verbunden. ${ }^{19}$

Darüber hinaus sind noch immer fast täglich Menschenrechtsverletzungen durch die Angriffe der internationalen Schutztruppen oder aufständische Gruppen zu verzeichnen, wobei die Letztgenannten für den Großteil der Opfer verantwortlich sind. ${ }^{20}$ Obwohl verschiedene internationale und nationale Menschenrechtsorganisationen zu mehr Rücksichtnahme auf Zivilisten aufgerufen haben, ${ }^{21}$ hat sich die Lage im letzten Jahr sogar noch verschlechtert. ${ }^{22}$

\section{Auswirkungen schwacher Rechtsstaatlichkeit auf die Sicherheitslage}

Die schwach ausgeprägte Rechtsstaatlichkeit wirkt sich aufgrund unterschiedlicher Faktoren negativ auf die Sicherheitslage aus: Das Versagen der Sicherheitskräfte bei der Herstellung der öffentlichen Ordnung bewirkt einen Anstieg der Kriminalitätsrate, insbesondere im Bereich des organisierten Verbrechens wie dem Drogenhandel. ${ }^{23}$ Doch auch die Zahl der Diebstähle, Raubüberfälle und Entführungen nimmt zu. ${ }^{24}$

Korruption und Parteilichkeit der Richter bewirken darüber hinaus, dass einflussreiche und vermögende Straftäter sich sicher sein können, für ihr Handeln nicht belangt zu werden, was sie dazu ermutigt, weitere Straftaten zu begehen. Für die Verletzungen von Menschenrechten wird ebenfalls niemand zur Verantwortung gezogen. Das gesamte Justizsystem, soweit überhaupt zugänglich, erweist sich daher momentan als ineffektiv und nicht fähig, für Gerechtigkeit im Land zu sorgen.

Diese Ungerechtigkeit steigert den Zorn der Bevölkerung auf das System und gefährdet dadurch dessen Stabilität. ${ }^{25}$ Die aufständischen Gruppen, insbesondere die Taliban, nutzen die an-

18 AIHRC Strategic Plan and Action Plan, 1389-1392 (2010- 2013), Part II: The Human Rights Situation in Afghanistan, S. 10; (http://www.aihrc.org.af/english/Eng_pages/Action_Plan_E/ Strategic_Action_Plans.pdf, 10. September lish/Eng_pages/Action_Plan_E/ Strategic__ction_Plans.pd, 10. September 2010).

19 Siehe United Nations Office of the High Commissioner for Human Rights "Human Rights Dimensions of Poverty in Afghanistan" (Poverty Report), Kabul, April 2010, S. 14

202009 starben 67 Prozent der bei Kampfhandlungen getöteten Zivilisten durch Handlungen der Aufständischen, 25 Prozent durch Angriffe der regierungsnahen Truppen oder internationalen Streitkräfte. Bei acht Prozent war nicht herauszufinden, welche Konfliktpartei die Ursache war (S.G. Chesser, „Afghanistan Casualites: Military Forces and Civilians“, S. 4; http://www.fas.org/sgp/ crs/natsec/R41084.pdf; 10. September 2010).

21 Neben der AIHRC war das unter anderem Oxfam International mit Unterstützung zahlreicher nationaler und internationaler NGOs.

222009 war das verlustreichste Jahr seit dem Sturz der Taliban 2001. Laut UNAMA wurden insgesamt 2412 Zivilisten getötet und mindestens 3566 verletzt (UNAMA Afghanistan Annual Report on Protection of Civilians in Armed Conflict, 2009, S. 1 ff. (http://unama.unmissions.org/Portals/UNAMA/ human\%20rights/Protection\%20of\%20Civilian\%202009\%20report\%20En glish.pdf, 10. September 2010)

23 Hierzu hat UNODC Afghanistan verschiedene Publikationen herausgegeben, siehe http://www.unodc.org/afghanistan/en/publications-and-reports.html (10.September 2010).

24 Eindruck aus aus persönlichen Gesprächen. Siehe auch Martin Patience „Afghanistan's Kidnapping Industry“, 16. September 2008, http://news.bbc. co.uk/2/hi/south_asia/7596224.stm (10. September 2010).

25 Siehe S. Carter und K. Clark (Fn 5), S. 28 ff. gesprochenen Missstände bewusst für ihre Propaganda ${ }^{26}$ und Rekrutierungen. ${ }^{27}$

Die Taliban hatten sich bereits während ihrer Herrschaft über das „Islamische Emirat Afghanistan“ als Verfechter von Gerechtigkeit und Sicherheit verstanden. Sie stellten klare Regeln auf, die es einzuhalten galt. Auch wenn diese teilweise fundamentale Menschenrechtsverletzungen darstellten, gelang es ihnen doch, eine gewisse Ordnung und Stabilität im Land herzustellen. Die drakonischen Strafen, die die Taliban eingeführt hatten, sorgten für einen Rückgang der Kriminalität und somit für ein gewisses Maß an Sicherheit, das viele Afghanen heute vermissen und zurücksehnen. ${ }^{28}$ Mittlerweile haben die Taliban in einem Teil der von ihnen kontrollierten Gebiete wieder ein eigenes Justizsystem eingerichtet. Es genießt einen besseren Ruf als die staatlichen Gerichte und wird als fair - im Sinne der Taliban-Ideologie - und effizient wahrgenommen. ${ }^{29}$

\section{Neuere Entwicklungen seit 2010}

Die Destabilisierung im Land und die Verschlechterung der Sicherheitslage haben insbesondere seit 2009 zu einem Umdenken der internationalen und nationalen Akteure geführt. Bezüglich des militärischen Einsatzes hat der ehemalige Oberbefehlshaber der internationalen Truppen, General McChrystal, eine neue Strategie verkündet, die insbesondere Rücksichtnahme auf die Zivilbevölkerung bei militärischen Angriffen versprach. ${ }^{30}$ Die Strategie soll auch unter McChrystal's Nachfolger General Petraeus weiter verfolgt werden. ${ }^{31}$

Auf der internationalen Kabul-Konferenz im Juli 2010 hat die afghanische Regierung die „National Priority Programs“ präsentiert, eine Reihe von Entwicklungsprogrammen, die zwischen 2010 und 2013 umgesetzt werden sollen. Damit beginnt eine neue Phase des seit November 2009 andauernden Kabul Process, in dem die afghanische Regierung gemeinsam mit Vertretern der Zivilgesellschaft und dem privaten Sektor die Führungsrolle bei der Implementierung von Entwicklungsprogrammen übernehmen soll. Die afghanische Regierung sieht sich ausdrücklich in der Pflicht, das Leben der afghanischen Bürger durch Armutsbekämpfung, die Etablierung von Recht und Ordnung und den Ausbau der Wirtschaft zu verbessern. ${ }^{32}$ Im Folgenden sollen die Programme „Law and Justice for all“ und „Human Rights and Civic Responsiblity“ kurz beschrieben und analysiert werden.

26 International Crisis Group „Taliban Propaganda: Winning the War of Words?" Asia Reports $N^{\circ} 158,24$. Juli 2008, S. 22 ff., http://www.crisisgroup.org/ / media/Files/asia/south-asia/afghanistan/ 158_taliban_propaganda___ winning_the_war_of_words.ashx (10. September 2010).

27 Sarah Ladbury und Cooperation for Peace and Unity "Testing Hypotheses on Radicalization in Afghanistan", Kabul, August 2009, S. 20 ff, 23.

28 Eindruck aus Gesprächen vor Ort. Siehe auch Ulrich Laduner „Die Stadt und ihre Mörder", http://www.zeit.de/2010/15/DOS-Afghanistan (10.Septebmer 2010).

29 Frank Ledwidge "Justice and Counter-Insurgency in Afghanistan: A Missing Link", The RUSI Journal, 154:1, S. 7.

30 Siehe "Commander's Initial Assessment" vom 30. August 2009.

31 Obama entlässt Oberbefehlshaber McChrystal (FAZ.Net: www.faz.net/s/Rub 0CCA23BC3D3C4C78914F85BED3B53F3C/Doc EB6697054555140CFA8805 C99029BF031 ATpl Ecommon Scontent.html; 10. September 2010).

32 Afghan National Development Strategy - Prioritization and Implementation Plan, Mid 2010 - Mid 2010, Volume I, S. 4 (http://www.mfa.gov.af/kcs/ ANDS\%20PIP\%20Vol\%201\%20-\%20English.pdf; 10.September 2010). 


\section{National Program for Law and Justice for Al/33}

Dieses Programm sieht im Wesentlichen die Reform der Justizinstitutionen, den Ausbau der Infrastruktur, die Überarbeitung bestehender sowie die Verabschiedung neuer Gesetze vor. Hierdurch soll die Effizienz der Justiz erhöht und ihr Ansehen in der Bevölkerung gesteigert werden. Durch die Schließung von Gesetzeslücken soll mehr Rechtssicherheit und -klarheit geschaffen werden. Auch das Sicherheitsproblem der Mitarbeiter des Justizsektors wird angesprochen; zu ihrem Schutz wird an die Aufstellung einer speziellen Polizeieinheit gedacht.

Die Umsetzung der in dem Programm vorgesehenen Maßnahmen trifft auf unterschiedliche Hindernisse und bedarf genau angepasster Strategien. Einige Beispiele sollen dies verdeutlichen. Eine Reihe der Maßnahmen des Programms, wie z.B. die bessere Ausstattung von Justizgebäuden oder die Reform von Arbeitsabläufen, müssten eigentlich in kürzester Zeit umsetzbar sein, deshalb ist es zunächst einmal verwunderlich, dass diese Vorschläge bereits in der National Justice Sector Strategy (NJSS) ${ }^{34}$ aus dem Jahr 2007 enthalten waren. An mangelnden finanziellen Mitteln dürfte es eigentlich nicht liegen, dass diese Missstände immer noch bestehen. Auch im Hinblick auf die Verwaltung und Organisation dieser Institutionen müssten die Mängel rasch zu finden sein, sodass die Durchführung einer umfassenden Reform eigentlich nicht zu schwierig sein dürfte.

Zur Fortbildung von Juristen gibt es bereits mehrere Programme in Afghanistan, die auch auf reges Interesse stoßen. Angesetzt werden müsste bereits bei der universitären Ausbildung. Momentan kann entweder an einer Sharia-Fakultät bzw. privaten Madrassa islamisches Recht studiert werden oder an einer JuraFakultät das staatliche Recht. Da das afghanische Recht jedoch sowohl islamisches als auch säkulares Recht beinhaltet, müssten die Lehrpläne der Fakultäten angeglichen werden. Dies ist bisher jedoch noch nicht in ausreichendem Maße geschehen.

Andere Reformprozesse benötigen wesentlich mehr Zeit, sie sollten überdies nicht überstürzt angegangen werden. Auch wenn bestehende Gesetzeslücken dringend geschlossen werden müssen, sollte umsichtig vorgegangen werden, denn zu groß ist die Gefahr, dass entweder der Gesetzgebungsprozess ungeordnet voranschreitet oder Gesetze sich gegenseitig widersprechen. In der Vergangenheit sind die Gesetze teilweise von ausländischen Organisationen überarbeitet bzw. von ihnen komplett entworfen worden, sie liegen zum Teil auch nur in englischer Sprache vor. Soweit Übersetzungen existieren, sind sie bisweilen fehlerhaft und für die Richter unverständlich, sodass die Gesetze nicht anwendbar sind. ${ }^{35}$

Fraglich ist des Weiteren, wann den Richtern tatsächlich so viel persönliche Sicherheit gewährleistet werden kann, dass sie ihre Urteile frei von äußeren Drohungen fällen können. Der Umsetzungsplan sieht hierfür einen Zeitraum von einem Jahr

33 ANDS Prioritization and Implementation Plan (Ebenda, Fn 32), Volume II, S. $57 \mathrm{ff}$ (http://www.mfa.gov.af/kcs/ANDS\%20PIP\%20Vol\%202\%20\%20English.pdf; 10. September 2010).

34 Siehe z.B. Justice \& Rule of Law Sector Strategy 1378 - 1391, Chapter 2, Goal 1: Improved Institutional Capacity to Deliver Sustainable Justice. Es handelt sich hierbei um ein an die Afghan National Development Strategy (ANDS) aus dem Jahr 2007 angegliedertes Programm.

35 Information aus persönlichen Gesprächen. vor. ${ }^{36}$ Allerdings richten sich Drohungen oftmals auch gegen Familienangehörige, was den Kreis der Schutzbedürftigen deutlich erweitert. Es ist außerdem fraglich, ob Polizeipräsenz das richtige Rezept für das Sicherheitsproblem ist. Manche Richter sind deshalb gefährdet, weil sie aufgrund ihrer Bestechlichkeit einen schlechten Ruf bei der Bevölkerung haben. ${ }^{37}$ Würden sie ihr Verhalten ändern, würde sich wohl auch ihre Sicherheitsgefährdung vermindern.

Das Programm sieht außerdem vor, den Zugang zur Justiz durch eine Verknüpfung der formellen und informellen Justizsektoren zu verbessern. Wie bereits ausgeführt, hat der formelle Sektor aufgrund von Korruption, fehlender Unparteilichkeit und seiner Ineffizienz einen schlechten Ruf bei der afghanischen Bevölkerung. Informelle Streitschlichtungsmechanismen, die stattdessen konsultiert werden, fällen ihre Entscheidungen in der Regel jedoch nicht auf Grundlage des geltenden afghanischen Rechts, sondern gemäß Stammesgesetzen wie etwa dem Paschtunwali oder einer zweifelhaften Auslegung des islamischen Rechts. Diese Entscheidungen verstoßen daher nicht selten gegen das nationale Recht, die afghanische Verfassung oder internationale Menschenrechtskonventionen. Neuere Bestrebungen, insbesondere von Seiten der USA und Großbritanniens, zielen daher auf eine Verknüpfung beider Sektoren ab. Dadurch soll einerseits der informelle Justizsektor der Überwachung durch die formellen Institutionen unterworfen werden, andererseits bleiben aber die traditionellen Streitschlichtungsmechanismen erhalten, die von der Bevölkerung offensichtlich bevorzugt werden. ${ }^{38}$

Eine Verknüpfung beider Sektoren hätte gleich mehrere Vorteile: zum einen könnte sie zu einer Entlastung der Gerichte führen, da viele Fälle von den traditionellen Mechanismen entschieden würden und das Ergebnis von einem Richter anschließend lediglich dokumentiert werden müsste. Dem großen Rückstau an Fällen, wie er momentan noch vorherrscht, würde entgegengewirkt. Weiterhin würde der Zugang zur Justiz erleichtert, insbesondere in solchen Gegenden, in denen es bislang keine Gerichte gibt.

Allerdings muss auch in den außergerichtlichen Verfahren gewährleistet werden, dass die Entscheidungen im Einklang mit der formellen Rechtsordnung stehen. Insbesondere ist $\mathrm{zu}$ bezweifeln, dass die Entscheidungsträger - zum Teil sind es Taliban - in den Provinzen des Landes, auf die die Regierung in Kabul so gut wie keinen Einfluss hat, das formelle Recht jemals akzeptieren werden. Dieser Prozess wird daher noch viele Jahre andauern, insbesondere auch deshalb, weil das Verhältnis zwischen formellem und informellem Sektor in jeder Region anders ausgestaltet ist. Eine pauschale Regelung für die angestrebte Verknüpfung zu finden dürfte daher schwierig sein und erfordert eine gründliche Analyse sämtlicher regionaler Besonderheiten. Dies wiederum ist aufgrund der angespannten Sicherheitslage momentan so gut wie unmöglich.

36 Prioritization and Implementation Plan, Volume II (Fn 33), S. 63.

37 Wie in dem oben beschriebenen Fall der Richter in Kandarhar (Fn 15)

38 Ausführlicher hierzu Julia Pfeiffer "Customary Law and Informal Justice in Afghanistan", Verwaltung und Recht in Übersee, 2010 (im Erscheinen). 


\section{Program for Human Rights and Civic Responsibilities ${ }^{39}$}

Dieses Programm sieht vor, das Bewusstsein für Menschenrechte, insbesondere Frauenrechte, sowohl innerhalb afghanischer staatlicher Institutionen als auch der Bevölkerung zu stärken. Da die große Bedeutung informeller Institutionen mittlerweile erkannt worden ist, sollen auch innerhalb dieser die Menschenrechte gefördert werden. Der Schutz von Menschenrechten ist jedoch ein heikles Thema in Afghanistan, denn sie werden als Erfindung des Westens angesehen und deshalb nicht vollständig akzeptiert. Allerdings ist ein umfassender Grundrechtskatalog in der Verfassung enthalten und von Vertretern des Volkes auch so verabschiedet worden. Zudem sind Menschenrechte dem islamischen Recht nicht fremd. Bildung beispielsweise spielt im Islam eine große Rolle, und zwar für beide Geschlechter. Auch die Rolle der Frau kann im islamischen Recht durchaus anders interpretiert werden, als dies von der afghanischen Gesellschaft derzeit getan wird.

Vielen Afghanen scheint darüber hinaus gar nicht bewusst zu sein, dass Menschenrechte einen Schutz der Bürger gegen den Staat bedeuten und genutzt werden können, um die persönliche Situation zu verbessern. Im afghanischen Rechtssystem gibt es zudem bislang nur wenige wirksame Rechtsmittel, um gegen Menschenrechtsverletzungen vorzugehen und es bedarf in dieser Hinsicht noch vieler Verbesserungen.

Das Programm greift auch das Problem der Straflosigkeit für Verbrechen auf, die seit dem Beginn des Konfliktes in Afghanistan, insbesondere während des Bürgerkriegs, begangen worden sind. Um einer solchen Straflosigkeit entgegenzuwirken, soll der bereits erwähnte Action Plan for Peace and Reconciliation in einer aktualisierten Version wieder eingeführt werden. Darüber hinaus sollte das Bewusstsein von Regierung, internationaler Gemeinschaft und Öffentlichkeit dafür geschärft werden, dass ein dauerhafter Frieden im Land nur erreicht werden kann, wenn Täter - auf der Basis rechtsstaatlicher Prinzipien - zur Verantwortung gezogen werden.

Eine Lösung für dieses Problem zu finden, wird allerdings nicht einfach sein. Während die einen Gerechtigkeit und eine Bestrafung der Täter verlangen, stehen für andere pragmatische Erwägungen im Vordergrund. Warlords haben weiterhin die Kontrolle über Teile des Landes oder besetzen wichtige Positionen in Parlament und Regierung. So wird befürchtet, dass eine strafrechtliche Verfolgung dieser Personen die Stabilität in den von ihnen kontrollierten Gebieten des Landes gefährden und die Lage dort eher noch verschlimmern würde. Es bleibt zunächst abzuwarten, wie die aktualisierte Version des Action Plans aussehen wird und welche Maßnahmen vorgesehen werden, um die Täter zur Verantwortung zu ziehen.

Denkbar zumindest sind auch andere Maßnahmen, um ein Mindestmaß an Gerechtigkeit im Land herzustellen. Dazu müsste die Justiz unterstützt von der Regierung verstärkt gegen Korruption und das organisierte Verbrechen vorgehen - bis in die Führungsspitzen des Staates hinein. Auch eine konsequente Strafverfolgung aller Täter würde die Straflosigkeit für einflussreiche Personen beseitigen und für mehr Gerechtigkeit

39 Siehe hierzu Prioritization and Implementation Plan, Volume II (Fn 33), S. $66 \mathrm{ff}$. im Land sorgen. Dazu müssten nicht nur Vetternwirtschaft und Korruption überwunden, sondern auch die Machtverhältnisse im Land neu geordnet werden. Da jedoch viele der Personen, die die nötigen Änderungen herbeiführen könnten, von den Konsequenzen selbst betroffen wären, ist eine solche Entwicklung wenig wahrscheinlich. Die staatlichen Entscheidungsträger ignorieren ganz offensichtlich ihre Verantwortung gegenüber der Bevölkerung; dass sie an der Verschlechterung der Situation in ihrem Land selbst beteiligt sind, scheint sie wenig zu stören. Eine wesentliche Verbesserung der Menschenrechtslage wird unter diesen Bedingungen ein langer Prozess sein.

\section{Schlussfolgerung}

Rechtsstaatlichkeit hat sich in Afghanistan bislang nicht ausreichend etablieren können. Die instabile Sicherheitslage und schwach ausgeprägte rechtsstaatliche Strukturen verstärken sich dabei gegenseitig.

Der Ansatz, der nun im „Kabul-Prozess“ verfolgt wird, zeigt, dass der afghanische Staat sich den Problemen und Zusammenhängen durchaus bewusst ist. Auch wenn die präsentierten Strategien und Programme in der Theorie gut klingen, so kann eine Verbesserung der Situation selbstverständlich nur erreicht werden, wenn ihre Umsetzung mit Nachdruck vorangetrieben wird.

Stabile Rechtsstaatlichkeit ist eine Grundvoraussetzung für den Fortschritt im Land. Jedoch müssen westliche Vorstellungen von einem Rechtsstaat in Afghanistan relativiert und traditionelle Streitbeilegungsmechanismen dürfen aufgrund ihrer Bedeutung nicht komplett außer Acht gelassen werden. Gleichzeitig muss den Missständen im formellen Rechtssektor konsequent entgegengewirkt werden, sonst wird dieser nicht über die Grenzen Kabuls hinaus etabliert werden können. Ein Mindestmaß an Gerechtigkeit in absehbarer Zeit herzustellen müsste möglich sein, wenn vor allem Korruption und Straflosigkeit der Kampf angesagt wird. Dies würde das Ansehen der Justiz wesentlich verbessern und zu mehr Akzeptanz des Rechtssystems bei der Bevölkerung führen. Um die momentanen Missstände abzustellen, bedarf es der Zusammenarbeit aller staatlichen Institutionen. Diese müssen sich ihrer Verantwortung gegenüber dem Volk jedoch erst noch bewusst werden.

Wann eine solche Veränderung spürbar stattfinden wird, ist momentan jedoch nicht einzuschätzen. Der Kabul-Prozess, der diese Veränderung zumindest in der Theorie vorbereitet, soll noch bis Mitte 2013 andauern. Ob es sich dabei um einen zu ambitionierten Zeitplan handelt, wird sich wohl erst in den nächsten Monaten herausstellen. Um zu beweisen, dass die afghanische Regierung es mit ihren Vorsätzen tatsächlich ernst meint, sollte sie mit der Umsetzung der vorgesehenen Programme unverzüglich beginnen. 